\title{
Analytical BER Performance Evaluation in SISO and MIMO Environments with SC-FDE Modulations and IB-DFE Receivers
}

\author{
Filipe Casal Ribeiro $^{1,4}$ - Rui Dinis ${ }^{2,4}$ - Francisco Cercas ${ }^{1,4}$. \\ Adão Silva ${ }^{3,4}$
}

Published online: 24 May 2017

(C) Springer Science+Business Media New York 2017

\begin{abstract}
This paper presents the analysis of the obtainment of the theoretical bit error rate (BER) performance in single-input-single-output and multiple-input-multiple-output systems with single-carrier with frequency-domain equalization modulations and iterative receivers based on the iterative block decision feedback equalization concept. Through the consideration of a Gaussian-based approach to obtain the BER performance, we present a simple and accurate model to improve such method by compensating the difference between the theoretical performance results and the ones obtained by simulation.
\end{abstract}

Keywords Theoretical BER performance $\cdot$ SISO $\cdot$ MIMO $\cdot$ SC-FDE · IB-DFE

\section{Introduction}

Block transmission techniques with cyclic prefix (CP) insertion and integrated with frequency-domain processing, such as orthogonal frequency division multiplexing (OFDM) [1] and single-carrier with frequency domain equalization (SC-FDE) [2], are proved to be the most suitable for broadband wireless systems. These techniques constitute, therefore, the primarily effective sources of anti-multipath systems, due to the trade-off offered between performance in multipath propagation and in signal processing involvement. OFDM and SC-FDE present near global processing requirements and performances. Nevertheless, the transmitter complexity is higher for OFDM and the receiver complexity

Filipe Casal Ribeiro

casalribeiro.filipe@gmail.com

1 ISCTE - Instituto Universitário de Lisboa, Lisbon, Portugal

2 FCT - Universidade Nova de Lisboa, Lisbon, Portugal

3 DETI - Universidade de Aveiro, Aveiro, Portugal

4 IT, Instituto de Telecomunicações, Lisbon, Portugal 
is higher for SC-FDE, which makes SC-FDE preferable for the uplink transmission while OFDM is interesting mainly for the downlink transmission. Moreover, the envelope fluctuations of single-carrier signals are much lower than the envelope fluctuations of OFDM signals with the same constellations [3,4]. The frequency-domain equalization performed in conjunction with block transmission systems is computationally more straightforward when comparing with the time-domain approach. This is due to the fact that operations on a block level involve an efficient FFT operation and a simple channel inversion processing. Usually a linear FDE equalizer is employed with SC-FDE techniques. The filtering can be performed through a zero forcing (ZF) or minimum meansquared error (MMSE) criterium, in which the MMSE presents better performances when compared with the ZF, due to the lower noise enhancement effects [7]. Although nonlinear equalizers are more complex than linear equalizers, it is known that they offer better performnce/complexity trade-offs [5]. The most promising nonlinear FDE is the iterative block decision feedback equalization (IB-DFE) [5, 6], which is an iterative FDE implemented in the frequency-domain [5].

This paper presents the bit error rate (BER) performance of IB-DFE receivers with SCFDE modulations schemes. These performance are usually obtained by means of lengthy Monte Carlo simulations and it would be desirable to analytically obtain the BER performance of IB-DFE receivers. Since the overall noise plus residual Inter-Symbol Interference (ISI) is approximately Gaussian at the FDE output, it can be obtained an estimate of the BER from the MSE at the FDE output for each iteration [8]. These BER estimates are very accurate for the linear FDE, however, they present a non-negligible error in the iterative FDE case and in this paper it is shown that the error is a function of the number of multipath propagation components, as well as the MSE. Ultimately, it is presented a method for estimating and compensating for the error of MSE-based BER performances, enhancing the accuracy of analytical BER estimates.

This paper is organized as follows: Sect. 2 describes the cellular scenarios and the iterative receiver design considered in this paper. Section 3 explains the Gaussian-based approach for obtaining the BER performance and details an improved method for enhancing the accuracy of the Gaussian model described previously. Section 4 concludes the paper.

Throughout the paper we will adopt the following notations: bold letters denote vectors and matrices; $\mathbf{x}^{*}, \mathbf{x}^{T}$ and $\mathbf{x}^{H}$ denote complex conjugate, transpose and Hermitian (complex conjugate transpose) of $\mathbf{x}$, respectively. $\mathbf{I}_{N}$ denotes a $N \times N$ identity matrix and $\mathbf{e}_{p}$ is an appropriate column vector with 0 in all positions except the $p$ th position that is 1 . The expectation of $x$ is denoted by $\mathbb{E}[x]$.

\section{System Characterization}

The system is characterized by the employment of a CP-assisted SC-FDE modulation scheme between $P$ MTs sharing the same physical channel that transmit to $R$ BSs. After removing the cyclic prefix, the received useful frequency-domain signal, at a certain BS $r$, is given by

$$
Y_{k}^{(r)}=\sum_{p=1}^{P} S_{k, p} H_{k, p}^{e p(r)}+N_{k}^{(r)} .
$$


Here, $\left\{S_{k, p} ; k=0,1, \ldots, N-1\right\}$ corresponds to the DFT of the time-domain data block, $\left\{s_{n, p} ; n=0,1, \ldots, N-1\right\}$, associated to the $p$ th MT $(p=1,2, \ldots, P)$, where constellation symbol $s_{n, p}$ is selected from the data according to a certain mapping rule (e.g., a QPSK constellation with Gray mapping), and with a $N$ symbols block size. The useful timedomain received block at the $r$ th $\mathrm{BS}$ is $y_{n}^{(r)}(r=1,2, \ldots, R)$, and the corresponding frequency-domain block is $Y_{k}^{(r)}=\operatorname{DFT}\left\{y_{n}^{(r)}\right\}$. Moreover, in (1), $N_{k}^{(r)}$ denotes the channel noise at the $r$ th antenna and the $k$ th frequency and $H_{k, p}^{e q(r)}=\xi_{p, r} H_{k, p}^{(r)}$, where $H_{k, p}^{(r)}$ corresponds to the channel frequency response between the $p$ th MT and the $r$ th BS, for the $k$ th frequency. The combined effects of power control and propagation implementations are weighted by the factor $\xi_{p, r}$. Hence, the average received power at the receiver is $\left|\xi_{p, r}\right|^{2}$, where it is assumed a normalized channel frequency response, with $\mathbb{E}\left[\left|H_{k, p}^{(r)}\right|^{2}\right]=1$.

When all the MTs and BSs contributions are taken into account the system can be regarded as a MIMO scheme, depicted in Fig. 1, and a matrix format of (1) can be defined as

$$
\mathbf{Y}_{k}=\mathbf{H}_{k}^{T} \mathbf{S}_{k}+\mathbf{N}_{k}
$$

where $\mathbf{Y}_{k}=\left[Y_{k}^{(1)}, \ldots, Y_{k}^{(R)}\right]^{T}, \mathbf{S}_{k}=\left[S_{k, 1}, \ldots, S_{k, P}\right]^{T}, \mathbf{N}_{k}=\left[N_{k}^{(1)}, \ldots, N_{k}^{(R)}\right]^{T}$ and

$$
\mathbf{H}_{k}^{T}=\left[\begin{array}{ccc}
H_{k, 1}^{e q^{(1)}} & \ldots & H_{k, P}^{e q^{(1)}} \\
\vdots & \ddots & \vdots \\
H_{k, 1}^{e q^{(R)}} & \cdots & H_{k, P}^{e q^{(R)}}
\end{array}\right]
$$

At the receiver, an DFE-type approach is taken into account (see Fig. 2). This structure is composed of two main filters, being the feedforward $F_{k}$ and the feedback $B_{k}$ blocks. Mainly, the $F_{k}$ coefficient is employed to equalize the channel and the $B_{k}$ to remove residual ISI and MAI (Multiple Access Interference), using the previous decisions for the ISI cancelation and computing a circuit reliability in the feedback loop to improve the estimation on symbols $\hat{s}_{n}$. Considering a SISO system, Fig. 3 presents the DFE concept applied to an iterative structure for block transmission techniques, i.e., an IB-DFE receiver. At the $i$ th iteration, the estimated symbols $\left\{\hat{s}_{n} ; n=0,1, \ldots, N-1\right\}$ are the hard decisions

Fig. 1 MIMO transmission scheme

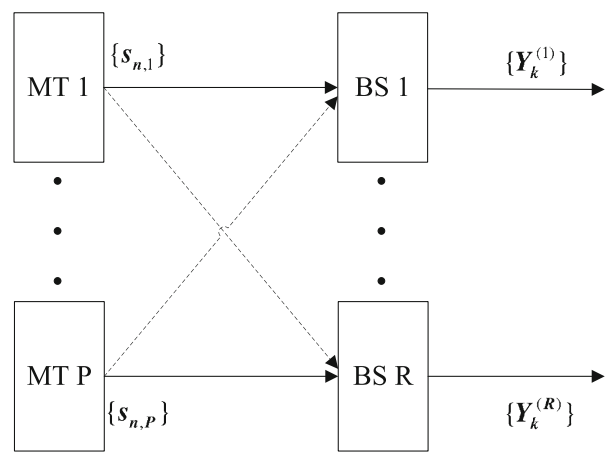


Fig. 2 DFE structure
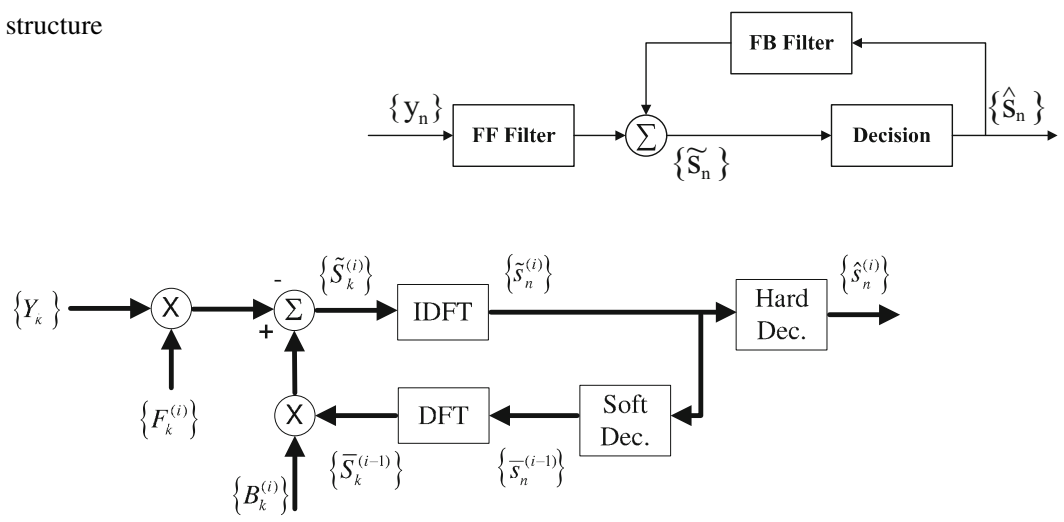

Fig. 3 IB-DFE receiver design for a SISO environment

of the time-domain detector output $\left\{\tilde{s}_{n} ; n=0,1, \ldots, N-1\right\}=$ IDFT $\left\{\tilde{S}_{k}^{(i)} ; k=0,1, \ldots, N-1\right\}$, where $\tilde{S}_{k}$ is given by

$$
\tilde{S}_{k}^{(i)}=F_{k}^{(i)} Y_{k}-B_{k}^{(i)} \bar{S}_{k}^{(i-1)},
$$

with $\left\{F_{k}^{(i)} ; k=0,1, \ldots, N-1\right\}$ denoting the feedforward coefficients and $\left\{B_{k}^{(i)} ; k=\right.$ $0,1, \ldots, N-1\}$ corresponding to the feedback coefficient. $\bar{S}_{k}^{(i-1)}$ is the DFT of the timedomain average values conditioned to the detector output $\left\{\bar{s}_{n}^{(i-1)} ; n=0,1, \ldots, N-1\right\}$ which is an estimate of the transmitted block obtained in the $(i-1)$ th iteration and used in the same estimate process in the current $i$ th iteration. Relating Fig. 3 with (2) the receiver design is expanded to Fig. 4.

For each iteration, the MTs are detected following a successive procedure, where the most updated output estimates of the transmitted data symbols associated to each MT are

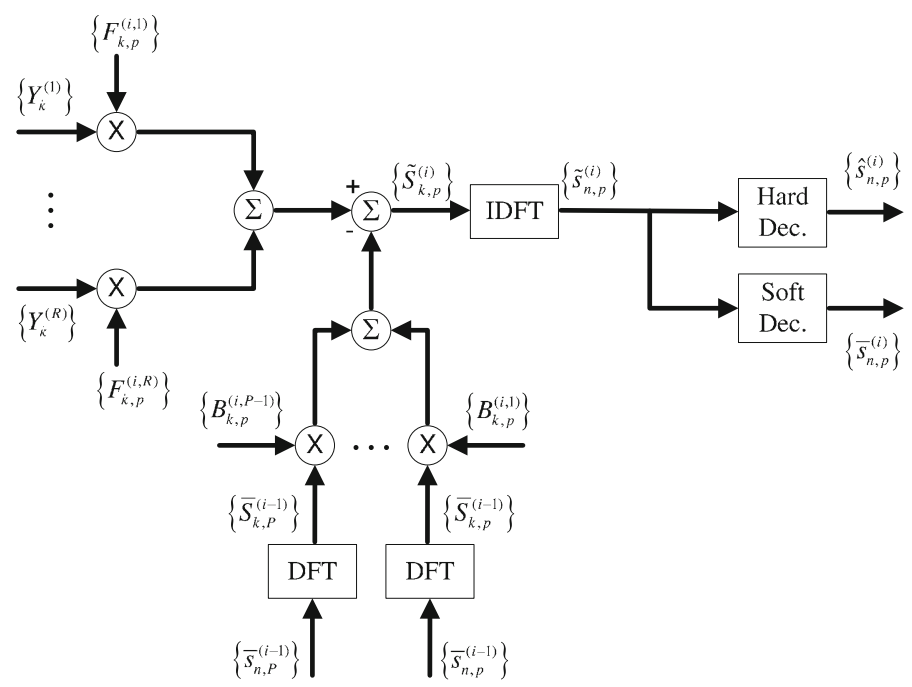

Fig. 4 IB-DFE receiver design for a MIMO environment 
used to cancel the corresponding residual interference. Therefore, the considered receiver can be regarded as an iterative Successive Interference Cancelation (SIC) scheme. Moreover, in this scenario, (4) can be expressed by

$$
\tilde{S}_{k, p}^{(i)}=\mathbf{F}_{k, p}^{(i)^{T}} \mathbf{Y}_{k}-\mathbf{B}_{k, p}^{(i)^{T}} \overline{\mathbf{S}}_{k, p}^{(i-1)},
$$

with $\quad \mathbf{F}_{k, p}^{(i)^{T}}=\left[F_{k, p}^{(i, 1)}, \ldots, F_{k, p}^{(i, R)}\right]^{T} \quad$ and $\quad \mathbf{B}_{k, p}^{(i)^{T}}=\left[B_{k, p}^{(i, 1)}, \ldots, B_{k, p}^{(i, P)}\right]^{T}$. The vector $\overline{\mathbf{S}}_{k, p}^{(i-1)}=\left[\bar{S}_{k, 1}^{(i)}, \ldots, \bar{S}_{k, p-1}^{(i)}, \bar{S}_{k, p}^{(i-1)}, \ldots, \bar{S}_{k, P}^{(i-1)}\right]^{T}$, where the block $\left\{\bar{S}_{k, p}^{(i)} ; k=0,1, \ldots, N-1\right\}$ is the DFT of the block of time-domain average values conditioned to the detector output $\left\{\bar{s}_{n, p}^{(i)} ; n=0,1, \ldots, N-1\right\}$ for user $p$ and iteration $i$. Clearly, the elements of $\overline{\mathbf{S}}_{k, p}^{(i-1)}$ are associated to the current iteration for MTs already estimated in this iteration and the previous iteration for the MT currently being detected, as well as the MTs that were not yet detected in the current iteration. For a normalized QPSK constellation set $\left(s_{n, p}= \pm 1 \pm j\right)$, the average values $\bar{s}_{n, p}$ correspond to

$$
\bar{s}_{n, p}=\tanh \left(\frac{L_{n, p}^{R e}}{2}\right)+j \tanh \left(\frac{L_{n, p}^{I m}}{2}\right),
$$

where

$$
\begin{aligned}
& L_{n, p}^{R e}=\frac{2}{\sigma_{n, p}^{2}} \operatorname{Re}\left\{\tilde{s}_{n, p}\right\}, \\
& L_{n, p}^{I m}=\frac{2}{\sigma_{n, p}^{2}} \operatorname{Im}\left\{\tilde{s}_{n, p}\right\},
\end{aligned}
$$

and

$$
\sigma_{n, p}^{2}=\frac{1}{2 N} \sum_{n^{\prime}=0}^{N-1}\left|\tilde{s}_{n^{\prime}, p}-s_{n^{\prime}, p}\right|^{2} \simeq \frac{1}{2 N} \sum_{n^{\prime}=0}^{N-1}\left|\tilde{s}_{n^{\prime}, p}-\hat{s}_{n^{\prime}, p}\right|^{2} .
$$

Moreover, $\sigma_{n}^{2}$ corresponds to the variance of the noise component and $\hat{s}_{n}$ correspond to the hard decisions, where $\operatorname{Re}\left\{\hat{s}_{n}\right\}=\operatorname{sign}\left(\operatorname{Re}\left\{\tilde{s}_{n}\right\}\right)$ and $\operatorname{Im}\left\{\hat{s}_{n}\right\}=\operatorname{sign}\left(\operatorname{Im}\left\{\tilde{s}_{n}\right\}\right)$.

\section{Theoretical BER Performance Evaluation}

Previous section described the parameters that define the system characterization in SISO and MIMO environments, as well as the iterative receiver based on the IB-DFE concept. In turn, this section deals with the theoretical analysis for obtaining the BER performance in a SC-FDE modulation scheme combined with IB-DFE receivers. A Gaussian-based approach is the starting point for the theoretical analysis for obtaining the BER performance.

At a given iteration, the coefficients $\mathbf{F}_{k, p}$ and $\mathbf{B}_{k, p}$ specify the iterative receiver's state. Furthermore, these coefficients are chosen to minimize Mean Squared Error (MSE) criterium, minimizing also the BER performance. Regarding a QPSK constellation and a Gray mapping scheme, the BER is given by 


$$
P_{b} \simeq Q\left(\sqrt{\frac{1}{M S E}}\right),
$$

where $Q(x)$ denotes the well known Gaussian error function and

$$
M S E=\frac{1}{N^{2}} \sum_{k=0}^{N-1} \Theta_{k, p},
$$

with

$$
\Theta_{k, p}=\mathbb{E}\left[\left|\tilde{S}_{k, p}-S_{k, p}\right|^{2}\right]=\mathbb{E}\left[\left|\mathbf{F}_{k}^{T} \mathbf{Y}_{k}-\mathbf{B}_{k}^{T} \overline{\mathbf{S}}_{k}-\mathbf{S}_{k}\right|^{2}\right],
$$

designating the MSE on the frequency-domain samples $\tilde{S}_{k, p}$. When the minimization of $\Theta_{k, p}$ is computed, the optimum values of the coefficients $\mathbf{F}_{k, p}$ and $\mathbf{B}_{k, p}$ are obtained and the BER performance is optimized. Regarding the iterative methodology of the described receiver in a MIMO scenario, it can be demonstrated (see appendix) that the optimum $\mathbf{F}_{k, p}$ and $\mathbf{B}_{k, p}$ are given, respectively, by

$$
\mathbf{F}=\kappa \Lambda \mathbf{H}^{H} \mathbf{e}_{p}
$$

and

$$
\mathbf{B}=\mathbf{H F}-\mathbf{e}_{p},
$$

with

$$
\boldsymbol{\Lambda}=\left(\mathbf{H}^{H}\left(\mathbf{I}_{P}-\mathbf{P}^{2}\right) \mathbf{H}+\frac{\sigma_{N}^{2}}{\sigma_{S}^{2}} \mathbf{I}_{R}\right)^{-1},
$$

and $\kappa$ selected to ensure that

$$
\frac{1}{N} \sum_{k=0}^{N-1} \sum_{r=1}^{R} F_{k, p}^{(r)} H_{k, p}^{e q(r)}=1 .
$$

Moreover, for a SISO system, at a given iteration, the optimum feedforward and feedback coefficients are expressed as

$$
F_{k}^{(i)}=\kappa \frac{H_{k}^{*}}{\frac{\sigma_{N}^{2}}{\sigma_{S}^{2}}\left(1-\left(\rho^{(i-1)}\right)^{2}\right)\left|H_{k}\right|^{2}}
$$

and

$$
B_{k}^{(i)}=\left(F_{k}^{(i)} H_{k}-1\right)
$$

with $\sigma_{S}^{2}$ and $\sigma_{N}^{2}$ denoting the signal and noise variance, respectively. The parameter $\rho$ designates a measure of the estimates reliability presented in the DFE loop, in order to deal with the error propagation phenomenon and is defined by 


$$
\rho=\frac{\mathbb{E}\left[\hat{s}_{n} s_{n}^{*}\right]}{\mathbb{E}\left[\left|s_{n}\right|^{2}\right]} .
$$

For QPSK constellations, $\rho=1-2 P_{b}$, which can be approximately

$$
\rho \approx \frac{1}{2 N} \sum_{n=0}^{N-1}\left(\left|\rho_{n}^{R e}\right|+\left|\rho_{n}^{I m}\right|\right),
$$

with

$$
\rho_{n}^{R e^{(i)}}=\tanh \left(\frac{\left|L_{n}^{R e^{(i)}}\right|}{2}\right)
$$

and

$$
\rho_{n}^{I^{(i)}}=\tanh \left(\frac{\left|L_{n}^{I m(i)}\right|}{2}\right) .
$$

For the previously described MIMO system with $P=2$ transmitting MTs and $R=2$ receiving BSs, Figs. 5 (1st MT) and 6 (2nd MT) illustrate the BER performance containing the simulated and theoretical approaches. The channel is a Rayleigh fading considering 8 rays multipath environment. Moreover, Figs. 7 and 8, for the 1st and 2nd MTs, respectively, show the same results, this time with 32 multipath rays. Both MTs have the same power $\left(\xi_{p, r}=\frac{\sqrt{2}}{2}\right)$ and the performance results are all compared with the Matched Filter Bound (MFB).

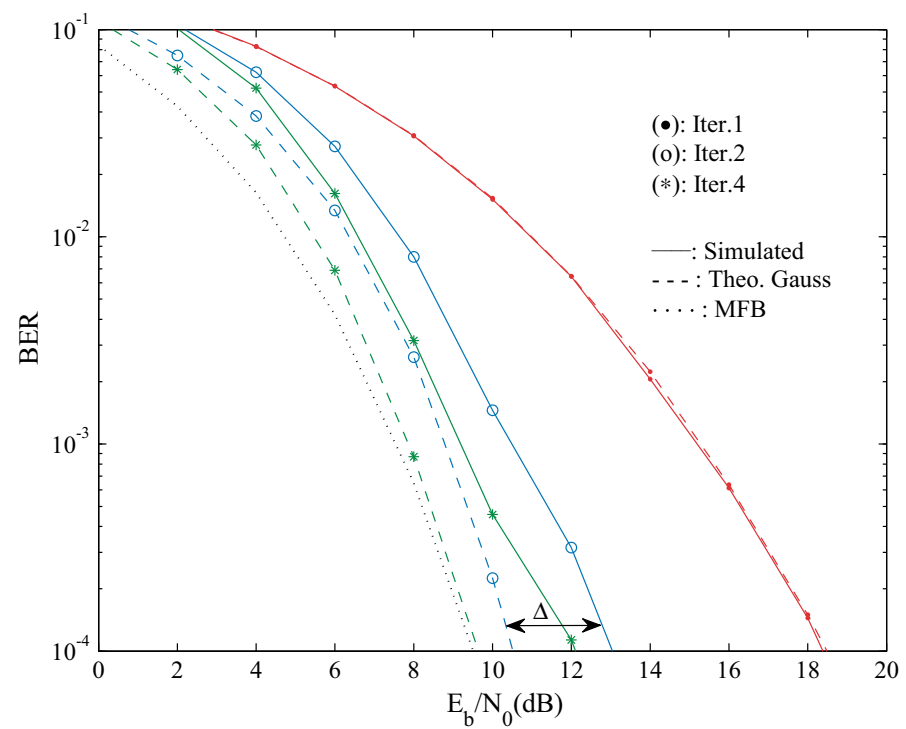

Fig. 5 BER performance with 8 rays in the multipath environment (1st MT) 


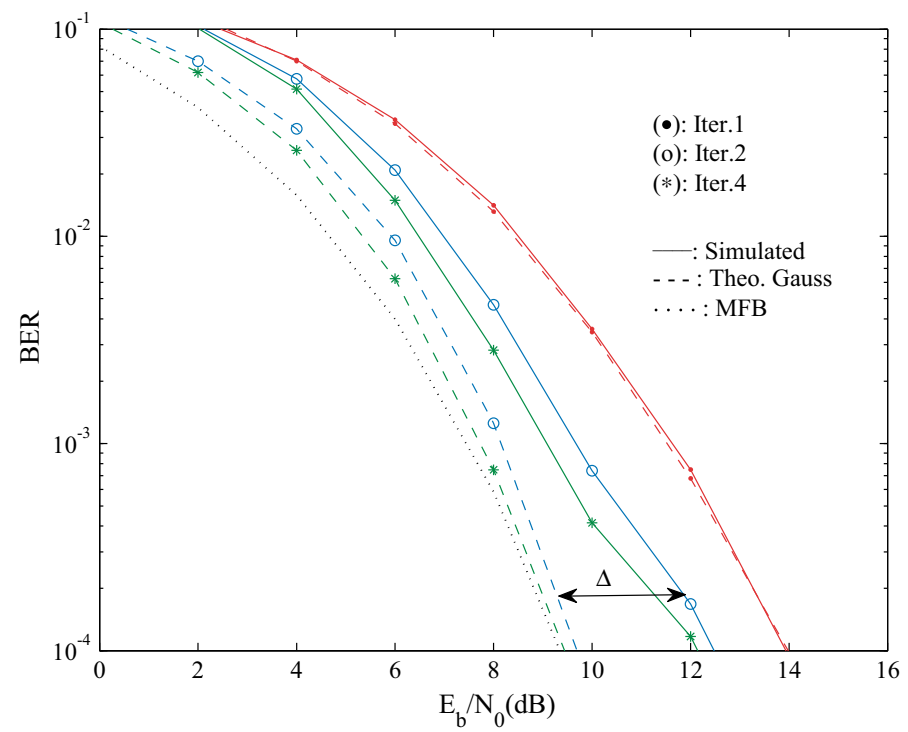

Fig. 6 BER performance with 8 rays in the multipath environment (2nd MT)

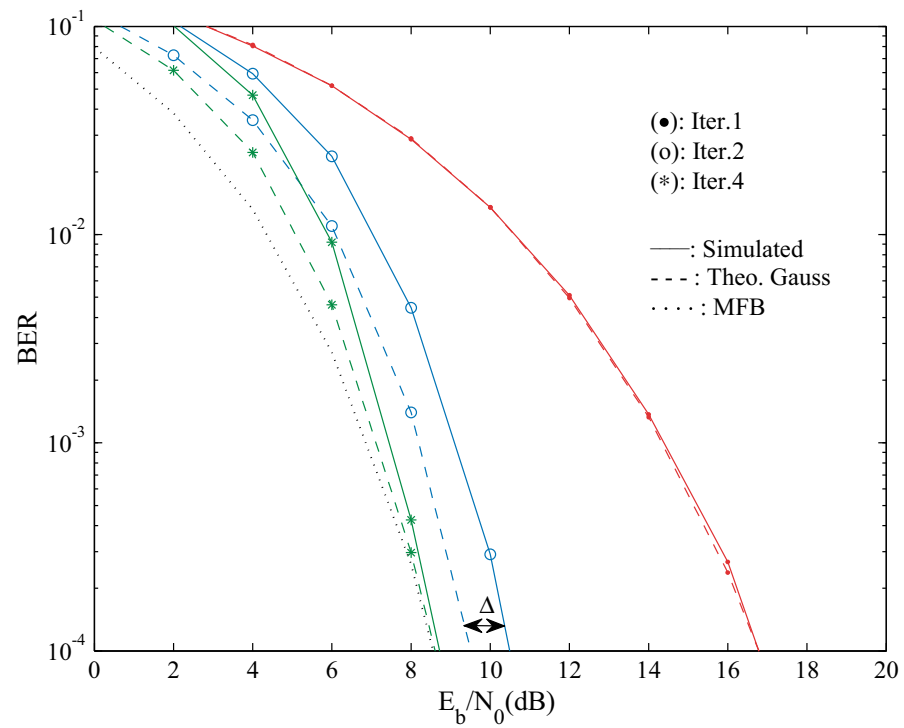

Fig. 7 BER performance with 32 rays in the multipath environment (1st MT)

The iterative receiver is characterized by a functionality of 4 iterations. It is easily observed that the performance increases with the number of iterations, in which by the time of the 4th iteration the performance is already close to the MFB. For simplicity purposes we only present iterations 1,2 and 4, since iteration 3 is very close to the 4 th iteration and doesn't add relevant information. Comparing the simulated and the analytical performances, there is a difference between them, corresponding to a value of $\Delta \mathrm{dB}$, except for 


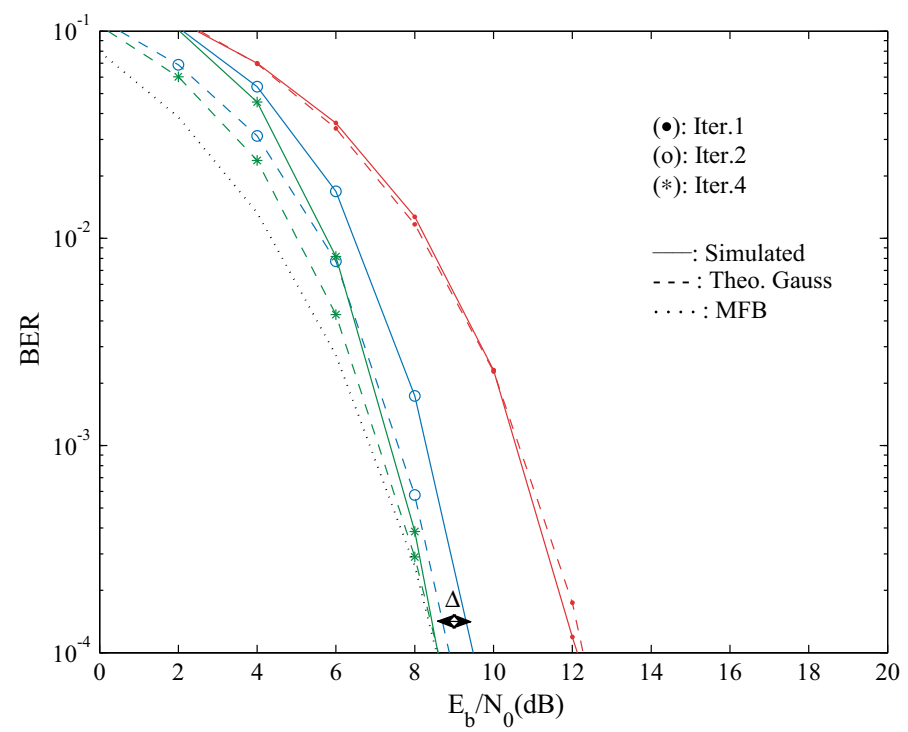

Fig. 8 BER performance with 32 rays in the multipath environment (2nd MT)

the linear FDE case. Moreover, the performances increases and approach the MFB as the number of rays in the multipath increases.

Relating the $\Delta$ with Eq. (9) it can be shown that

$$
P_{b} \simeq Q\left(\sqrt{\frac{1}{\Delta M S E}}\right)
$$

and the correct compensation of the $\Delta$ factor comes from understanding its behavior as a function of the number of rays in the multipath. Figure 9 shows, for both MTs, the $\Delta$ values for the 2 nd iteration with $8,16,32,64,128$ and 256 rays. We considered the $\Delta$ curves that correspond to the widely recognized reference values in the BER performance: $10^{-2}, 10^{-3}$ and $10^{-4}$. Analyzing Fig. 9, the $\Delta$ results presents a decreasing exponential behavior, that can be written as

$$
f(x)=a e^{-b x}+c,
$$

where $x$ indicates the number of rays considered in the multipath. Figs. 10 (1st MT) and 11 (2nd MT) illustrate the curve fitting with the approximation used by (22), demonstrating its accuracy. The optimum values that define the decreasing exponential for each BER reference are emphasized in Table 1 . In order to compensate the $\Delta$ factor, it is necessary to correctly perform its fitting. Therefore, the parameters $a, b$ and $c$, which are part of the approximation must be computed as well. Firstly, it is important to understand that we want to relate $\Delta$ with the BER evaluation and in turn with its corresponding MSE. To do so, we can use expression (9), and the BER values of $10^{-4}, 10^{-3}$ and $10^{-2}$ and indicate that their MSE corresponds to 0.07, 0.1041 and 0.1890 respectively. Figures 12 and 13 illustrates the approximation performed regarding the three parameters that constitute the $\Delta$ exponential likeness, for the 1st and 2nd MTs, respectively. After computing the fitting 


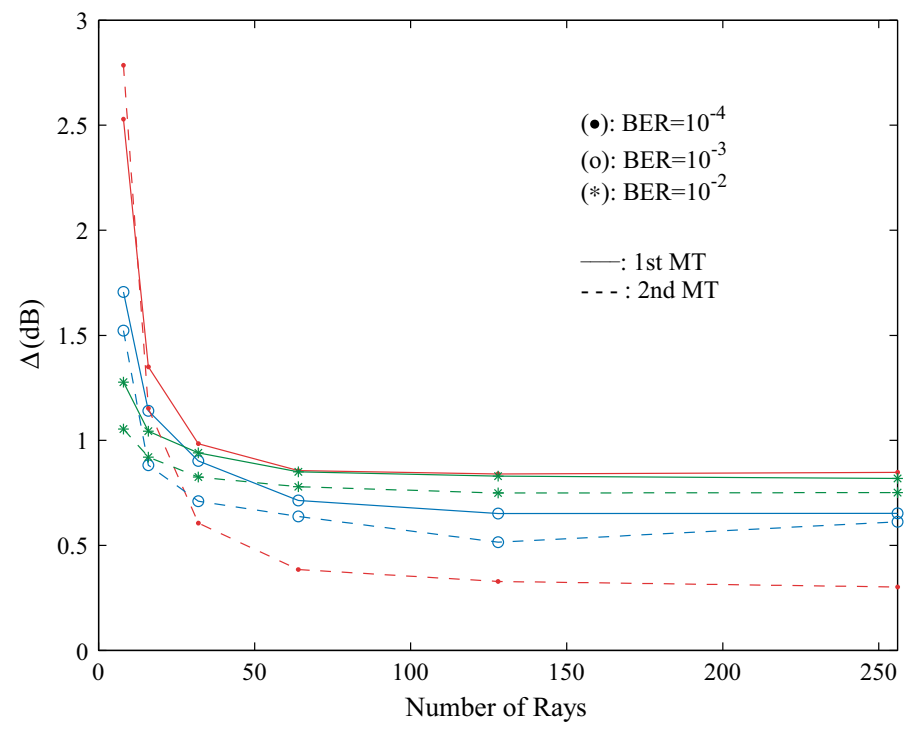

Fig. $9 \Delta$ behavior for the 2nd iteration and BER values of $10^{-4}, 10^{-3}$ and $10^{-2}$ (1st MT and 2nd MT)

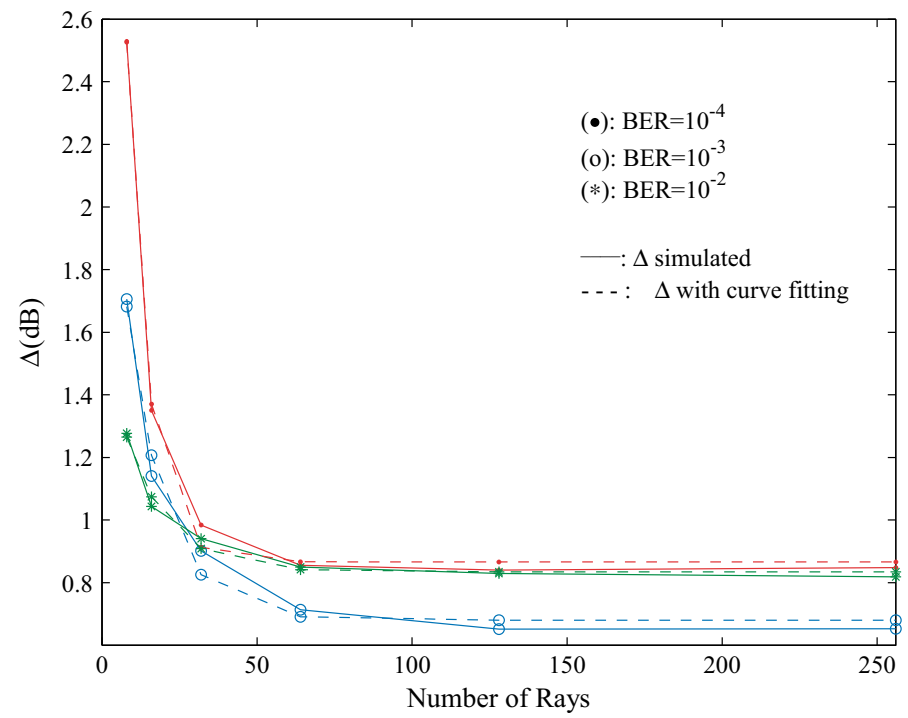

Fig. $10 \Delta$ behavior for the 2 nd iteration and BER values of $10^{-2}, 10^{-3}$ and $10^{-4}$ with curve fitting (1st MT) process for the $a, b$ and $c$ parameters of both MTs, the expressions that define the parameters, as a function of the MSE, are given by

$$
a(M S E)=48.12 e^{-31.79 M S E}+0.2442,
$$




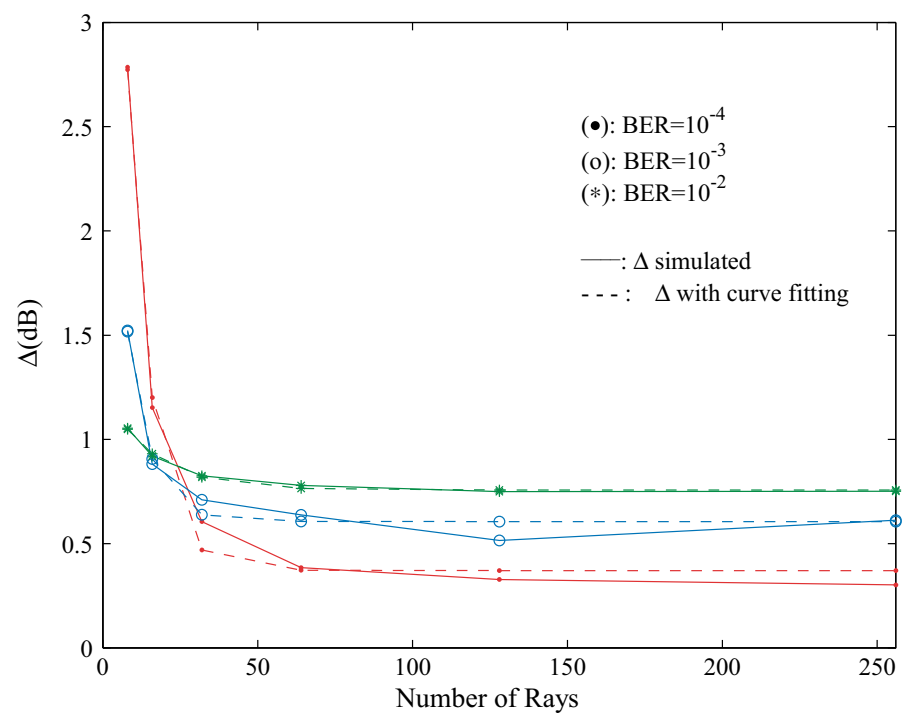

Fig. $11 \Delta$ behavior for the 2 nd iteration and BER values of $10^{-2}, 10^{-3}$ and $10^{-4}$ with curve fitting (2nd MT)

Table 1 Optimum values $a$, $b$ and $c$ for the 2nd iteration

\begin{tabular}{llll}
\hline 1 st MT & $\mathrm{a}$ & $\mathrm{b}$ & $\mathrm{c}$ \\
\hline $10^{-4}$ & 5.4664 & 0.1490 & 0.8664 \\
$10^{-3}$ & 1.9060 & 0.0804 & 0.6802 \\
$10^{-2}$ & 0.7734 & 0.0731 & 0.8343 \\
\hline $2 \mathrm{nd} \mathrm{MT}$ & $\mathrm{a}$ & $\mathrm{b}$ & $\mathrm{c}$ \\
\hline $10^{-4}$ & 6.9560 & 0.1329 & 0.3709 \\
$10^{-3}$ & 2.7700 & 0.1389 & 0.6059 \\
$10^{-2}$ & 0.4893 & 0.0646 & 0.7571 \\
\hline
\end{tabular}

$$
b(M S E)=0.7036 e^{-28.29 M S E}+0.04994
$$

and

$$
c(M S E)=1.452 M S E+0.625,
$$

for the 1st MT and

$$
\begin{gathered}
a(M S E)=44.33 e^{-26.57 M S E}+0.03952, \\
b(M S E)=-0.04405 M S E+0.1206
\end{gathered}
$$

and 


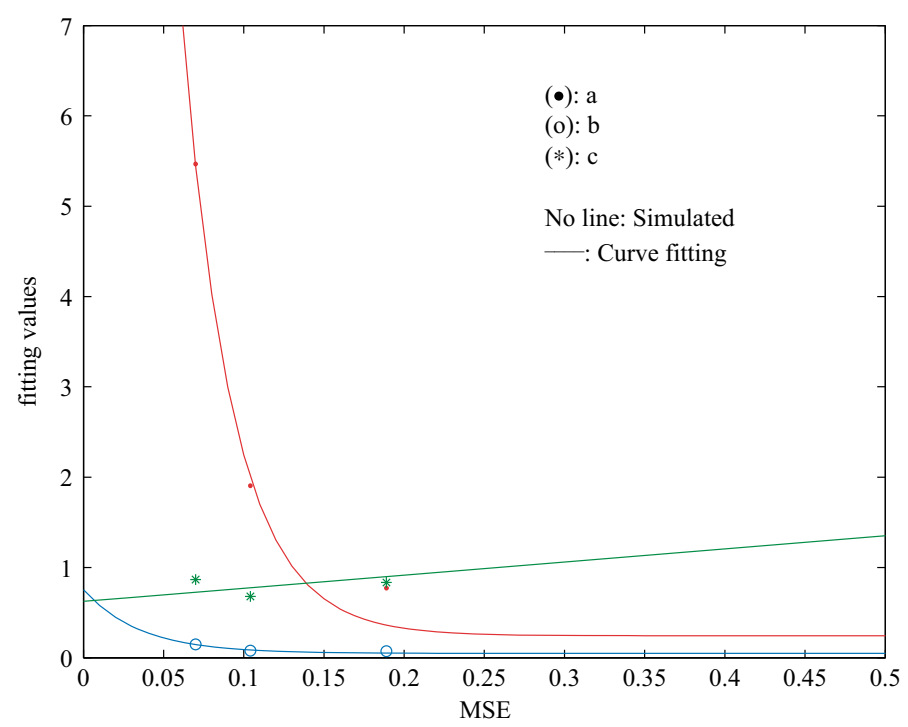

Fig. 12 Fitting of the $\Delta$ exponential approximation parameters $a, b$ and $c$, for the 2nd iteration (1st MT)

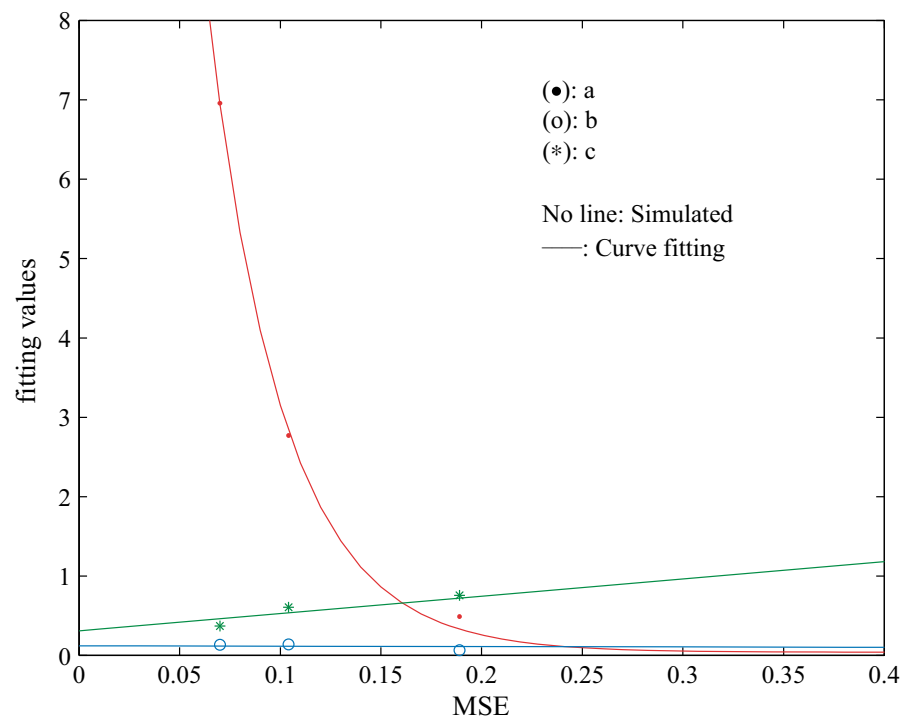

Fig. 13 Fitting of the $\Delta$ exponential approximation parameters $a, b$ and $c$, for the 2nd iteration (2nd MT)

$$
c(M S E)=2.177 M S E+0.3102,
$$

for the 2nd MT.

With all the exponential approximation parameters defined, it is possible, in a similar fashion to correctly compensate the $\Delta$ differences for iterations 3 and 4 , even though in these cases the value of $\Delta$ tends to be inferior when compared to the $\Delta$ in the 2nd iteration. The performances with the $\Delta$ correction in a multipath with 8 rays are shown in Figs. 14 


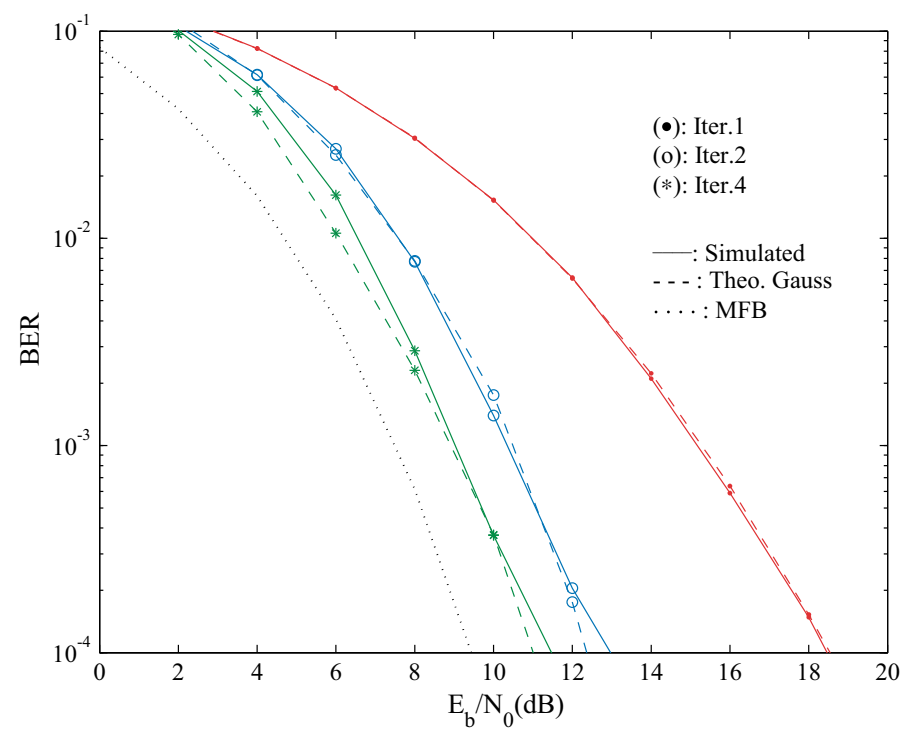

Fig. 14 BER performance with $\Delta$ correction and 8 rays in the multipath (1st MT)

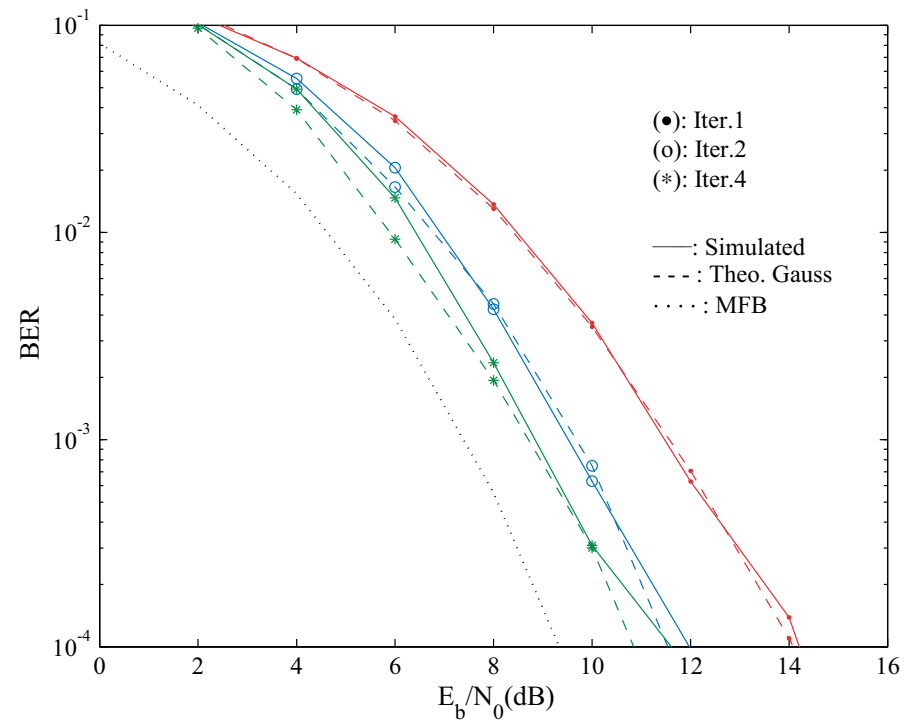

Fig. 15 BER performance with $\Delta$ correction and 8 rays in the multipath (2nd MT)

and 15 , for the 1st and 2nd MTs, respectively. The scenario with 32 rays is illustrated in Figs. 16 (1st MT) and 17 (2nd MT).

The method described for improving the theoretical BER performance obtainment corresponds to a MIMO system. Nevertheless, the same process can be straightforwardly applied to a SISO system. Hence, Figs. 18 and 19 illustrate the performance results for a 


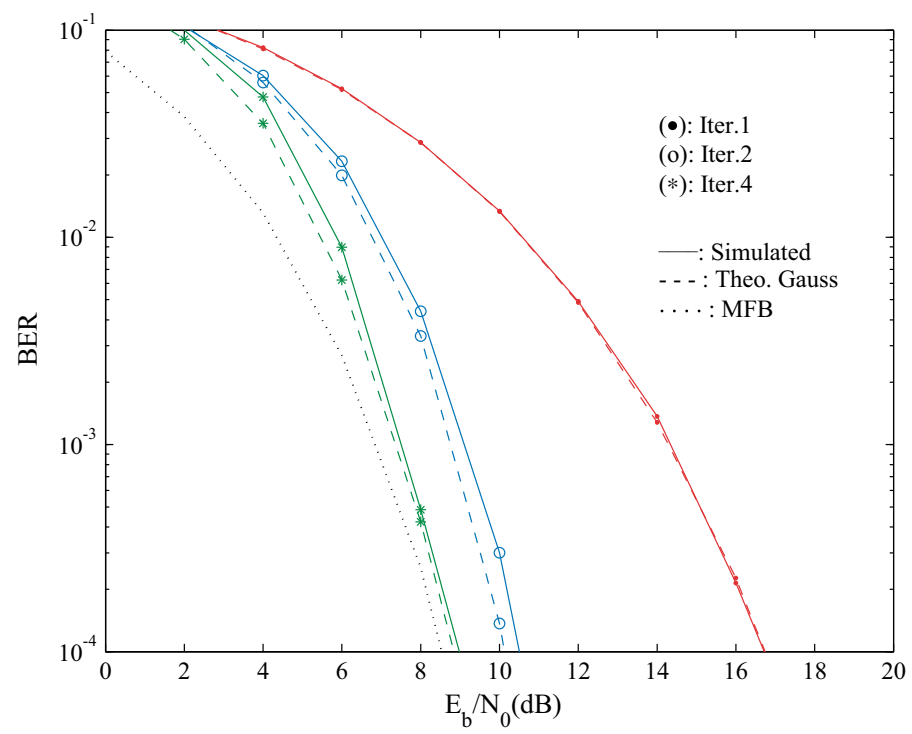

Fig. 16 BER performance with $\Delta$ correction and 32 rays in the multipath (1st MT)

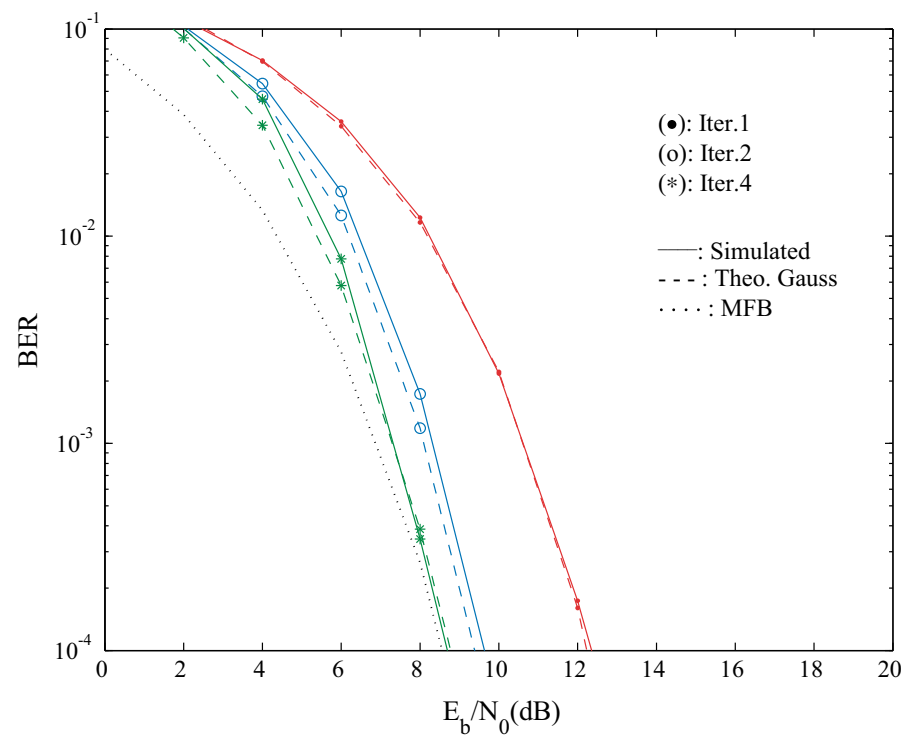

Fig. 17 BER performance with $\Delta$ correction and 32 rays in the multipath (2nd MT)

SISO system with 8 and 32 multipath rays, respectively. Moreover, Figs. 20 and 21 conclude the $\Delta$ correction process for Figs. 18 and 19 with the same approach as previously mentioned, where the conclusions are the same as in the MIMO system. 


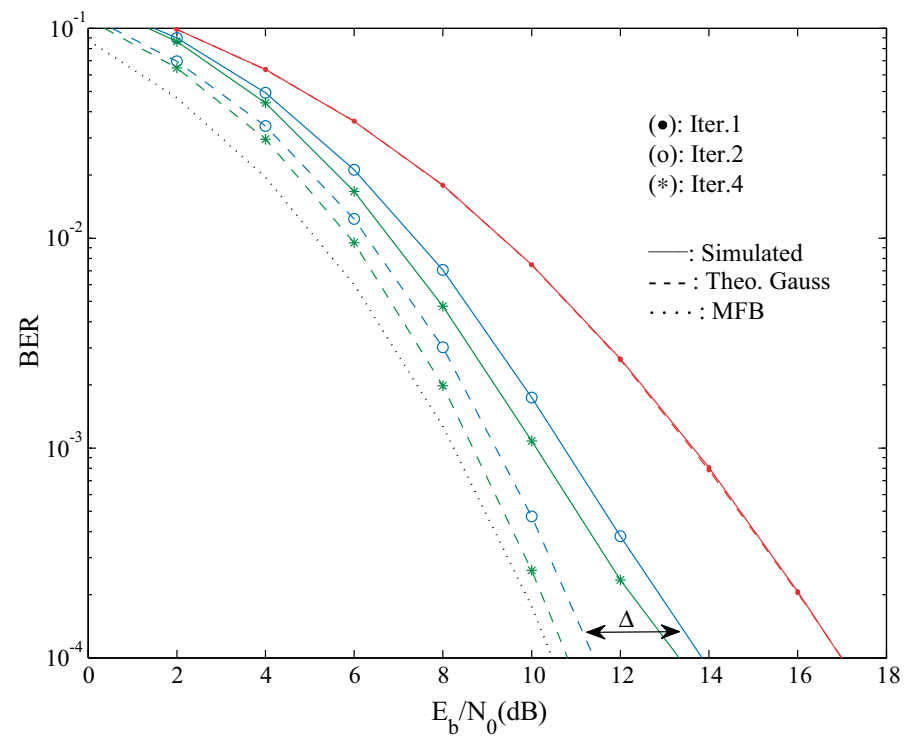

Fig. 18 BER performance with 8 rays in the multipath environment

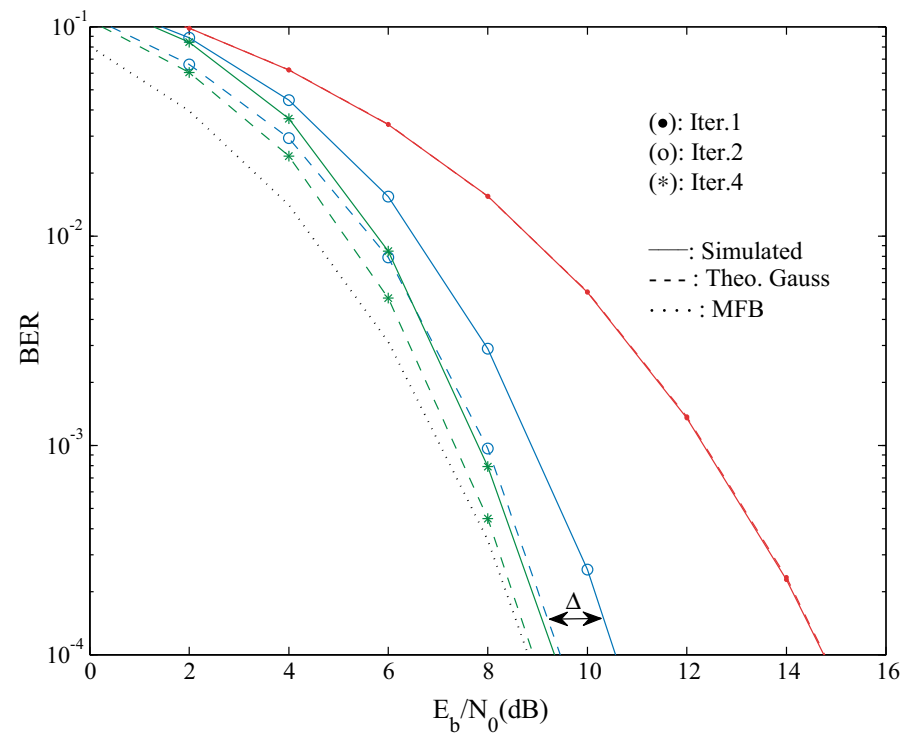

Fig. 19 BER performance with 32 rays in the multipath environment

\section{Conclusions}

This paper concerned the evaluation of analytically obtaining the BER performance in a transmission scheme with SC-FDE modulations and IB-DFE receivers for SISO and MIMO systems. It is presented a method for analytically obtaining the BER performance 


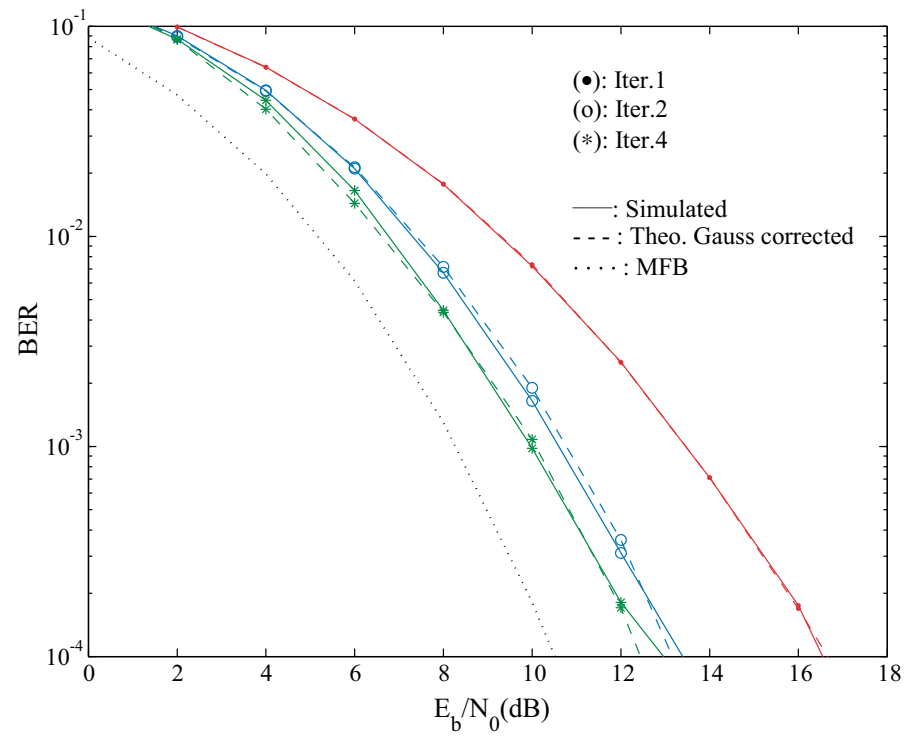

Fig. 20 BER performance with $\Delta$ correction and 8 rays in the multipath environment

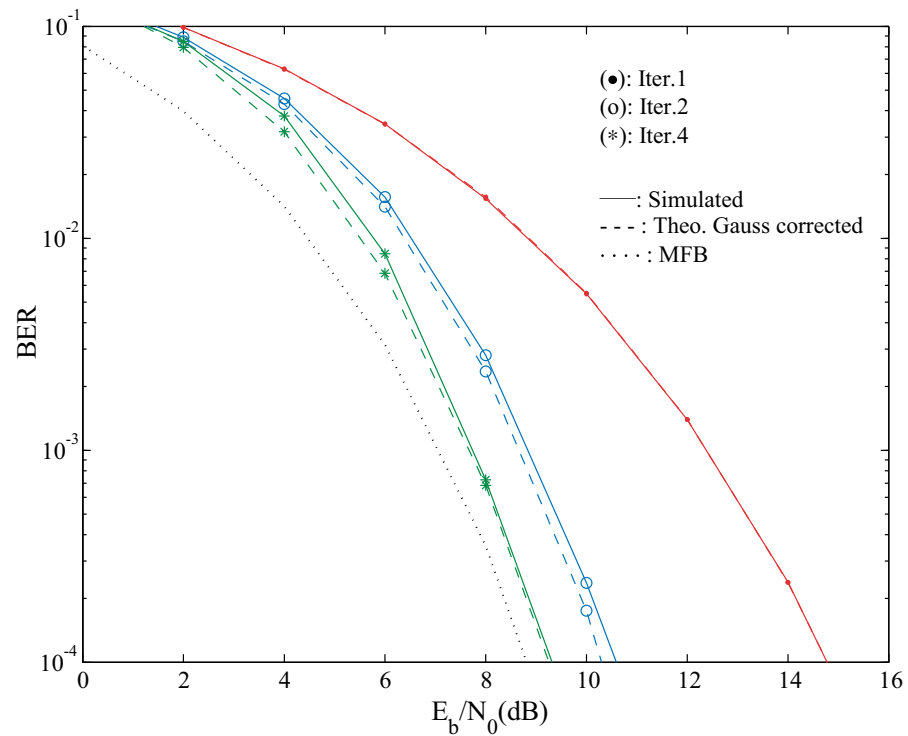

Fig. 21 BER performance with $\Delta$ correction and 32 rays in the multipath environment

by improving a Gaussian-based approach for its purpose. The method that allows the improvement of the theoretical approach is based on the compensation of the difference, designated by $\Delta$, between the simulated and theoretical BER performance results. The results show that this method is precise and the $\Delta$ compensation is accurate. 
Acknowledgements This work was partially supported by the FCT (Fundação para a Ciência e a Tecnologia) under Projects PEst-OE/EEI/LA0008/2013, HETCOP, ADIN (PTDC/EEI-TEL/2990/2012), CoPWIN (PTDC/EEI-TEL/1417/2012), UID/EEA/50008/2013 and Grant SFRH/BD/87524/2012.

\section{Appendix}

Considering a QPSK constellation with a Gray mapping rule, the bit error rate (BER) can be expressed as

$$
\begin{gathered}
P_{b}=Q\left(\sqrt{\frac{1}{M S E}}\right), \\
M S E=\frac{1}{N^{2}} \sum_{k=0}^{N-1} \Theta_{k, p},
\end{gathered}
$$

with

$$
\Theta_{k, p}=\mathbb{E}\left[\left|\tilde{S}_{k, p}-S_{k, p}\right|^{2}\right]=\mathbb{E}\left[\left|\mathbf{F}_{k}^{T} \mathbf{Y}_{k}-\mathbf{B}_{k}^{T} \overline{\mathbf{S}}_{k}-\mathbf{S}_{k}\right|^{2}\right]
$$

By assuming that $\bar{S}_{k, p} \simeq \rho_{p} \hat{S}_{k, p}$ [9], with $\hat{S}_{k, p}$ denoting the frequency-domain samples associated with the symbols' hard decision. Moreover, $\hat{S}_{k, p} \approx \rho_{p} S_{k, p}+\Delta_{k, p}$ [10], which means that $\bar{S}_{k, p} \approx \rho_{p}^{2} S_{k, p}+\rho_{p} \Delta_{k, p}$, and $\mathbb{E}\left[\left|\bar{S}_{k, p}\right|^{2}\right]=\left|\rho_{p}\right|^{2} \mathbb{E}\left[\left|\hat{S}_{k, p}\right|^{2}\right]=\left|\rho_{p}\right|^{2} \mathbb{E}\left[\left|S_{k, p}\right|^{2}\right]$. On the other hand, $\Delta_{k, p}=\left[\Delta_{k, 1}, \ldots, \Delta_{k, P}\right]^{T}$, is a mean zero error vector for $P$ MTs, with its elements uncorrelated to $S_{k, p}$ and between them, meaning that $\mathbb{E}\left[\left|\Delta_{k, p}\right|^{2}\right]=\left(1-\left|\rho_{p}\right|^{2}\right) \mathbb{E}\left[\left|S_{k, p}\right|^{2}\right]$. In matrix format, $\quad \overline{\mathbf{S}}_{k} \simeq \mathbf{P}^{2} \mathbf{S}_{k}+\mathbf{P} \boldsymbol{\Delta}_{k} \quad$ and $\mathbf{P}=\operatorname{diag}\left(\rho_{1}, \ldots, \rho_{P}\right)$.

By expanding the square in (31) and noting that the noise and data components are uncorrelated and have zero mean, it can be easily shown that $\Theta$ is given by

$$
\begin{aligned}
\Theta= & \mathbf{F}^{H} \mathbf{R}_{\mathbf{Y}} \mathbf{F}+\mathbf{B}^{H} \mathbf{R}_{\overline{\mathbf{S}}, \overline{\mathbf{S}}} \mathbf{B} \\
& +\mathbf{R}_{S}-2 \operatorname{Re}\left\{\mathbf{F}^{H} \mathbf{R}_{\mathbf{Y}, S_{p}}\right\} \\
& +2 \operatorname{Re}\left\{\mathbf{B}^{H} \mathbf{R}_{\overline{\mathbf{S}}, S_{p}}\right\}-2 \operatorname{Re}\left\{\mathbf{B}^{H} \mathbf{R}_{\mathbf{S}, \overline{\mathbf{Y}}} \mathbf{F}\right\} .
\end{aligned}
$$

For the sake of simplicity, the dependence on the subcarrier and user indexes, with the exception of the $S_{p}$ factor, were dropped from in (32) and following equations. The different correlation matrices of (32) are

$$
\begin{gathered}
\mathbf{R}_{Y}=\mathbb{E}\left[\mathbf{Y}^{*} \mathbf{Y}^{T}\right]=\mathbf{H}^{H} \mathbf{R}_{S} \mathbf{H}+\mathbf{R}_{N} \\
\mathbf{R}_{\overline{\mathbf{S}}, \overline{\mathbf{S}}}=\mathbb{E}\left[\overline{\mathbf{S}}^{*} \overline{\mathbf{S}}^{T}\right]=\mathbf{P}^{2} \mathbf{R}_{S} \\
\mathbf{R}_{\mathbf{Y}, S_{p}}=\mathbb{E}\left[\mathbf{Y}^{*} S_{p}\right]=\mathbf{H}^{H} \mathbf{R}_{S} \mathbf{e}_{p} \\
\mathbf{R}_{\overline{\mathbf{S}}, S_{p}}=\mathbb{E}\left[\overline{\mathbf{S}}^{*} S_{p}\right]=\mathbf{P}^{2} \mathbf{R}_{S} \mathbf{e}_{p} \\
\mathbf{R}_{\overline{\mathbf{S}}, \mathbf{Y}}=\mathbb{E}\left[\overline{\mathbf{S}}^{*} \mathbf{Y}\right]=\mathbf{P}^{2} \mathbf{R}_{S} \mathbf{H},
\end{gathered}
$$

with $\mathbf{R}_{S}=\mathbb{E}\left[\mathbf{S}^{*} \mathbf{S}^{T}\right]=2 \sigma_{S}^{2} \mathbf{I}_{P}$ and $\mathbf{R}_{N}=\mathbb{E}\left[\mathbf{N}^{*} \mathbf{N}^{T}\right]=2 \sigma_{N}^{2} \mathbf{I}_{R}$, being the correlation matrices of $\mathbf{S}$ and $\mathbf{N}$, respectively. 
Clearly, the bit error probability will be minimized if we minimize the MSE at each subcarrier $\Theta_{k, p}$. In order to obtain the minimization of the MSE we subject it to the condition

$$
\gamma_{p}=\frac{1}{N} \sum_{k=0}^{N-1} \sum_{r=1}^{R} F_{k, p}^{(r)} H_{k, p}^{e q(r)}=1,
$$

and apply the gradient of the Lagrange function to (31). Hence, the Lagrange function is defined as

$$
J=\Theta_{k, p}+\lambda\left(\gamma_{p}-1\right),
$$

where the optimum coefficients $\mathbf{F}_{k, p}$ and $\mathbf{B}_{k, p}$ being the solution for the system of equations

$$
\left\{\begin{array}{l}
\nabla_{\mathbf{F}} J=0 \\
\nabla_{\mathbf{B}} J=0 \\
\nabla_{\lambda} J=0
\end{array}\right.
$$

Therefore,

$$
\left\{\begin{array}{c}
\nabla_{\mathbf{F}} J=0 \Leftrightarrow \mathbf{R}_{\mathbf{Y}} \mathbf{F}-\mathbf{R}_{\mathbf{Y}, S_{p}}-\mathbf{R}_{\overline{\mathbf{S} Y}}^{H} \mathbf{B}+\lambda \mathbf{H}^{H} \mathbf{e}_{p}=0 \\
\nabla_{\mathbf{B}} J=0 \Leftrightarrow \mathbf{R}_{\overline{\mathbf{S}}, \mathbf{S}} \mathbf{B}+\mathbf{R}_{\overline{\mathbf{S}}, S_{p}}-\mathbf{R}_{\overline{\mathbf{S}}, \mathbf{Y}} \mathbf{F}=0 \\
\nabla_{\lambda} J=0 \Leftrightarrow \gamma_{p}=1 .
\end{array}\right.
$$

After some straightforward manipulations we obtain

$$
\mathbf{F}=\kappa \boldsymbol{\Lambda} \mathbf{H}^{H} \mathbf{e}_{p}
$$

and

$$
\mathbf{B}=\mathbf{H F}-\mathbf{e}_{p},
$$

with

$$
\boldsymbol{\Lambda}=\left(\mathbf{H}^{H}\left(\mathbf{I}_{P}-\mathbf{P}^{2}\right) \mathbf{H}+\frac{\sigma_{N}^{2}}{\sigma_{S}^{2}} \mathbf{I}_{R}\right)^{-1},
$$

and $\kappa$ selected to ensure that $\gamma_{p}=1$, in order to have a normalized FDE with $\mathbb{E}\left[\tilde{s}_{n, p}\right]=s_{n, p}$.

\section{References}

1. Cimini, L. J. (1985). Analysis and simulation of a digital mobile channel using orthogonal frequency division multiplexing. IEEE Transactions on Communications, 33(7), 665-675.

2. Sari, H., Karam, G., \& Jeanclaude, I. (1994). An analysis of orthogonal frequency-division multiplexing for mobile radio applications. In Vehicular technology conference (Vol. 3, pp. 1635-1639).

3. Gusmão, A., Dinis, R., Conceicão, J., \& Esteves, N. (2000). Comparison of two modulation choices for broadband wireless communications. In Vehicular technology conference proceedings (Vol. 2, pp. 1300-1305).

4. Falconer, D., Ariyavisitakul, S. L., Benyamin-Seeyar, A., \& Eidson, B. (2002). Frequency domain equalization for single-carrier broadband wireless systems. IEEE on Communications Magazine, 40(4), 58-66. 
5. Benvenuto, N., Dinis, R., Falconer, D., \& Tomasin, S. (2010). Single carrier modulation with nonlinear frequency domain equalization: An idea whose time has ComeAgain. Proceedings of the IEEE, 98(1), 69-96.

6. Benvenuto, N., \& Tomasin, S. (2005). Iterative design and detection of a DFE in the frequency domain. IEEE Transactions on Communications, 53(11), 1867-1875.

7. Proakis, J., \& Salehi, M. (2007). Digital communications (5th ed.). New York: Mc-Graw Hill.

8. Dinis, R., Silva, P., \& Gusmão, A. (2007). IB-DFE receivers with space diversity for CP-assisted DSCDMA and MC-CDMA systems. European Transactions on Telecommunications, 18(7), 791-802.

9. Gusmão, A., Torres, P., Dinis, R., \& Esteves, N. (2007). A turbo FDE technique for reduced-CP SCbased block transmission systems. IEEE Transactions on Communications, 55(1), 16-20.

10. Dinis, R., Gusmão, A., \& Esteves, N. (2003). On broadband block transmission over strongly frequency-selective fading channels. In Proceedings of the Wireless.

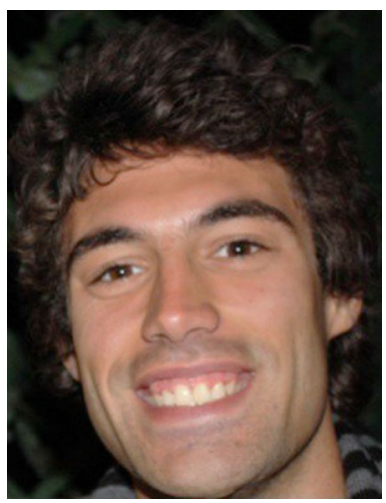

Filipe Casal Ribeiro received the M.S. degree in Telecommunications and Computer Science Engineering from ISCTE-Lisbon University Institute, Lisbon, Portugal in 2012. He is currently working towards the $\mathrm{Ph} . \mathrm{D}$. degree in Information Science and Technology in ISCTE-Lisbon University Institute, Lisbon, Portugal and in the Wireless Communications group of Telecommunications Institute, Lisbon, Portugal, since 2013. His research interests include wireless communications systems and frequency-domain equalization issues in MIMO environments.

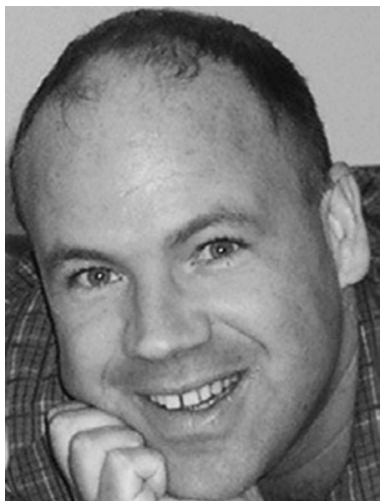

Rui Dinis received the Ph.D. degree from Instituto Superior Técnico (IST), Technical University of Lisbon, Portugal, in 2001. From 2001 to 2008 he was a Professor at IST. Since 2008 he is teaching at FCT-UNL (Faculdade de Ciências e Tecnologia da Universidade Nova de Lisboa). He was a researcher at CAPS/IST (Centro de Análise e Processamento de Sinais) from 1992 to 2005 ; from 2005 to 2008 he was researcher at ISR/IST (Instituto de Sistemas e Robótica); in 2009 he joined the research center IT (Instituto de Telecomunicações). He has been involved in several research projects in the broadband wireless communications area. His main research interests include modulation, equalization, synchronization and channel estimation. 

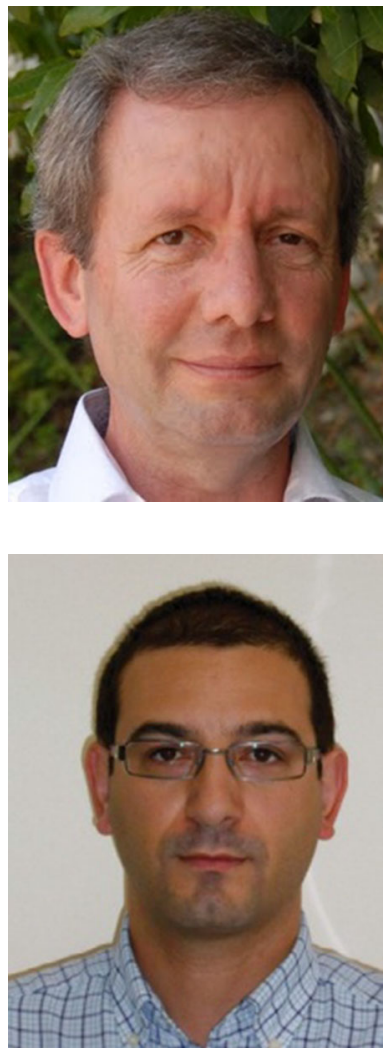

Francisco Cercas has more than 34 years of professional experience including more than 30 years of university teaching, 15 at IST and 16 at ISCTE-IUL where he is a Full Professor and the President of the Scientific Council. He is author and co-author of a new class of codes, TCH (Tomlinson, Cercas, Hughes), one patent, 4 book chapters, 14 journal papers, more than 150 conference papers and several research reports, 5 Ph.D.s theses supervision completed, as well as many MsC's and Final Year's Projects at both IST and ISCTE-IUL. He is Senior Member of IEEE, Coordinator of the Specialization in Telecommunications of Ordem dos Engenheiros since 2014 and Vice-coordinator of that Engineering Society between 2008 and 2013. He has been a researcher at CAPS, INESC, Satellite Centre of the University of Plymouth (UK) and Instituto de Telecomunicações, where he is presently a Director of the IT Lisbon pole). His areas of interest include satellite communications, location and positioning, modulation and coding theory, mobile communications and related topics.

Adão Silva received the M.Sc. and Ph.D. degrees in electronics and telecommunications from the University of Aveiro in 2002 and 2007, respectively. He is currently an Assistant Professor in the Department of Electronics, Telecommunications and Informatics of the University of Aveiro, and a researcher at the Instituto de Telecomunicações. He has participated in several national and European projects, namely ASILUM, MATRICE, and 4MORE within the ICT programme, and the FUTON and CODIV projects with the FP7 ICT. He has led several research projects in the broadband wireless communications area at the national level. He has been a member of the TPC of several international conferences. His research interests include multiuser MIMO, multicarrier based systems, cooperative networks, precoding, and multiuser detection. 